\title{
Thoracic paravertebral block for breast surgery in a pregnant woman -A case report-
}

\author{
Sang Il Park, Yoon-Hee Kim, Kyu Cheol Han, Sun Yeul Lee, Ann Misun Youn, Seok Hwa Yoon, and \\ Youn Hee Choi
}

Department of Anesthesiology and Pain Medicine, Chungnam National University School of Medicine, Daejeon, Korea

\begin{abstract}
Non-obstetrical surgery during the first trimester is stressful to both the mother and the fetus. Anesthesiologists are also stressed, not only because of the effects of surgery itself, but also because of the uncertain influences of anesthesia thrown upon on the fetus. The authors present a case of breast surgery successfully performed on a woman 8 weeks pregnant requiring removal of breast abscess by the application of thoracic paravertebral block without any complications. Thoracic paravertebral block may be a safe anesthetic method for non-obstetric surgery during early pregnancy. (Korean J Anesthesiol 2010; 59: S73-S76)
\end{abstract}

Key Words: Breast surgery, Pregnancy, Thoracic paravertebral block.

When preoperative pregnancy test are performed on childbearing aged women, $0.3-1.2 \%$ are shown to be positive [1]. Cases requiring non-obstetrical surgery during pregnancy is approximately $0.12 \%$ [2] and thus very low. However, when surgery is required, it becomes very stressful not only to the child bearer but also to the clinician. Anesthesia may cause physiological changes in the mother inducing teratogenic effects and fetal asphyxia, preterm labor, etc, to the fetus. It has been reported that the possibility of inducing deformity by clinically used anesthetics is almost nil; nonetheless, in cases undergoing surgery within 8 weeks of pregnancy, physiological changes of the mother caused by surgery and anesthesia may induce deformity, due to fetal organogenesis during that period. It is reported that if surgery is required during pregnancy, it is better to postpone it until the 2nd trimester if possible and preferably, perform regional anesthesia rather than general anesthesia [3].

The authors present a case of breast surgery successfully performed on a woman 8 weeks pregnant requiring removal of breast abscess by the application of thoracic paravertebral block (TPVB) without any complications.

\section{Case Report}

A 29-year-old female was under follow up observation at a local clinic for a mass in her left breast palpated 6 months prior to admission. Mass size increased due to pregnancy and showed inflammatory findings. She was thus transferred to our

Received: June 8, 2010. Revised: 1st, June 21, 2010; 2nd, June 29, 2010. Accepted: August 3, 2010.

Corresponding author: Yoon-Hee Kim, M.D., Department of Anesthesiology and Pain Medicine, Chungnam National University School of Medicine, 55, Munhwa-ro, Jung-gu, Daejeon 301-747, Korea. Tel: 82-42-280-7840, Fax: 82-42-280-7968, E-mail: yhkim0404@cnu.ac.kr (c) This is an open-access article distributed under the terms of the Creative Commons Attribution Non-Commercial License (http:// creativecommons.org/licenses/by-nc/3.0/), which permits unrestricted non-commercial use, distribution, and reproduction in any medium, provided the original work is properly cited. 
hospital for surgical treatment. Gestational age on admission was 8 weeks. In her presurgical breast ultrasonography, in the area $4 \mathrm{~cm}$ from the ten o'clock direction of the left nipple an approximately $4 \times 3 \mathrm{~cm}$ sized mass without any distinct boundaries thought to be an abscess was detected. In her past medical history, specific findings were denied and undetected. The mass was deeply located close to the fascia of the pectoralis muscle, thus difficult to operate by local anesthesia. Pretreatments were not given. In the operating room, it was decided that TPVB be performed because it would affect the fetus less than general anesthesia. The consent of the patient was obtained after through explanation of the anesthetic method. Basic monitoring equipments were attached to the patient. Initial blood pressure was $120 / 70$ $\mathrm{mmHg}$ and pulse rate 82 beats per minute. Other specific findings were undetected. The patient took a sitting position with an assistant was positioned in front of her. She was asked to lower her head and lean against the chest of the assistant. After marking the $\mathrm{C} 7$ spinous process (SP) to the T1 SP with a skin marker, each mark of the T1 SP to the T5 SP was marked again horizontally $2.5 \mathrm{~cm}$ left of the initial mark. After marking the areas, her skin was prepared with povidone iodide solution and subsequently, $1.5 \mathrm{ml}$ of $2 \%$ lidocaine was injected into each marked point subcutaneously. Afterwards, using five 20 gauge Tuohy needles, the skin of each point was pricked vertically. If the tip of the needle touched the transverse process, the needle was retreated and advanced by changing directions toward the head. The paravertebral space was assessed through a loss of resistance technique, and then the site was fixed. Each of the needles was installed in the marked site from the T1 SP to the $\mathrm{T} 5 \mathrm{SP}$ where a total of $20 \mathrm{ml}$ of $0.5 \%$ ropiacaine, $4 \mathrm{ml}$ each,

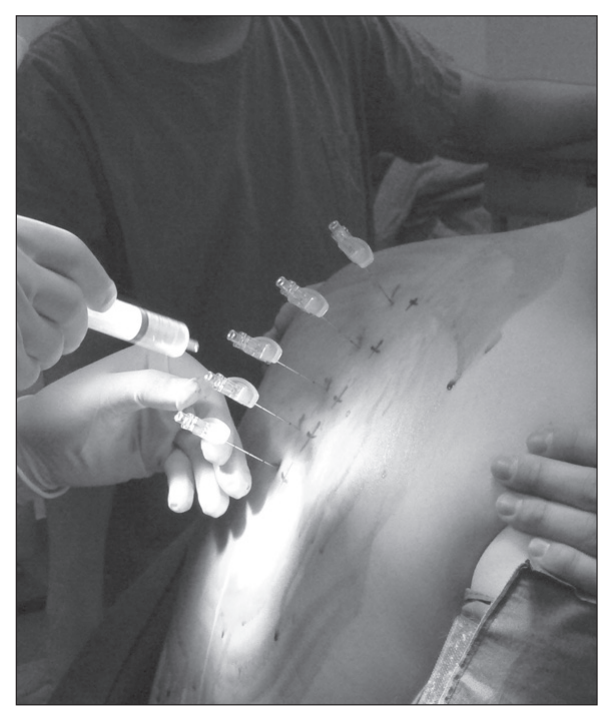

Fig. 1. After inserting 20 gauge Tuohy needles into each level, from $\mathrm{T} 1-\mathrm{T} 5,0.5 \%$ ropivacaine was injected. was injected. The needles were then removed (Fig. 1). Sterile dressing was performed on all areas injected with the drug. The patient then took the supine position, and blood pressure and pulse rate were $100 / 55 \mathrm{mmHg}$ and 100 , respectively. Other specific findings were undetected and the patient did not portray any particular symptoms. The blockage range evaluated by an ice test of the area from T1 to T10. During surgery, the patient presented with severe anxiety and requested to be put asleep, and so propofol (Fresofol ${ }^{\circledR}$, Fresenius Kabi, Austria), a pregnancy risk category $B$ drug, was injected using a syringe pump (Pilote anesthesie IS, Fresenius vial S.A, France) at the rate of $90 \mu \mathrm{g} / \mathrm{kg} / \mathrm{min}$. Total anesthesia time was 60 minutes and the total operation time was 40 minutes. After surgery, she was transferred to a recovery room for 45 minutes in order to detect any pain or other special findings she might experience. After transferring to a ward, where she also did not experience any pain, further analgesics were not administered.

In the ultrasonography performed the day after surgery, fetal heart beat was $180 \mathrm{bpm}$, and gestational age measured by crown-to-rump length was 8 weeks +5 days. The patient was treated with cefotiam $1 \mathrm{~g}$ /day through intravenous infusion for 4 days and was discharged without any complications.

\section{Discussion}

In cases performing surgery for non-obstetrical problems during pregnancy, maternal death, miscarriages, elective termination, and delivery induced by surgical procedure may be induced. For the fetus, fetal death, prematurity, and major birth defects may be induced. Particularly in cases undergoing surgery during the 1st trimester, the incidence of major malformation has been reported to be approximately $3.9 \%$. Maternal death rate is very low. However, during the entire pregnancy, the incidence of miscarriage or fetal death has been reported to be $5.8 \%$, and the risk raised to $10.5 \%$ during the 1st trimester. It has been reported that cases of selective termination due to anxiety of the birth of deformed babies were approximately $1.3 \%$ [4].

In cases which the patient must receive surgery unavoidably, safety of the mother and the fetus associated with anesthesia and surgery becomes an important issue. According to the standard labeling of drugs used in pregnancy which was organized in 1979, they are classified in 5 categories. Based on the research data conducted on animals and humans, the Food and Drug Administration classifies them as follows: $1 \mathrm{~A}$, Controlled studies have shown no risk; 1 B, No evidence of risk found in human beings; $1 \mathrm{C}$, A risk cannot be ruled out; $1 \mathrm{D}$, Positive evidence of risk exists; $1 \mathrm{X}$, The drug is contraindicated in pregnancy [5]. In animal experiments, nitrous oxide induces resorption and anomaly of the fetus [6]. In another 
experiments, inhalation anesthesia excluding nitrous oxide did not exert teratogenic effects. Studies reported that in humans exposed to sevoflurane at $0.2 \mathrm{ppm}$ concentration for 8 hours, sister chromatid exchange occurred more than in cases without exposure by $30 \%$ [7]. Intravenous anesthetics like meperidine, propofol, and ketamine belong to category B. Fentanyl has been reported to lack teratogenicity; nonetheless, it exerts embryocidal effects and is classified as category C. It is reported that diazepam rapidly penetrates the placenta and accumulates in the fetus, and exposure to it during pregnancy is associated with cleft palate. Different from diazepam, however, midazolam has been reported to lack teratogenicity but their pharmacological reaction is similar with diazepam and thus classified as category D and not recommended during the 1st trimester [8]. Drugs that can be used safely during pregnancy is limited. A prospectively controlled study cannot be conducted on humans due to ethical issues, and so it is indeed impossible to assess drug effects on the human fetus.

Regional anesthesia (RA) is safer than general anesthesia $[2,3]$. In regional anesthesia, less systemic absorption of drugs occur than general anesthesia and local anesthetics is classified as category B [5]. Thus, if surgical area or methods are appropriate, regional anesthesia can be performed safely. Nevertheless, during RA, rapid hemodynamic changes caused by sympathetic block may induce adverse effects on the fetus, and so appropriate precautions must be taken.

RA performed during breast surgery include thoracic epidural block (TEB), thoracic paravertebral block (TPVB), breast block, and intercostal nerve block. TEB and TPVB are generally performed.

TPVB is carried out by administering local anesthetics to the paravertebral space in the thoracic vertebra and inducing ipsilateral somatic and sympathetic nerve blockade of several levels of the thoracic dermatome [9]. The thoracic paravertebral space (TPVS) is a wedge-shaped space, its base formed by the posterolateral aspect of the vertebral body, intervertebral disc, and intervertebral foramen. The anterolateral wall is formed by the parietal pleura, and the posterior wall is formed by the superior costotransverse ligament. The lateral wall consists of the intercostal space and the intervertebral foramen, and the medial wall is consisted of the epidural space. The contralateral paravertebral space is connected to the prevertebral space. Techniques including the loss of resistance technique utilizing air in assessing the location, and also pressure measurement techniques and the methods using fluoroscopy [9] are known. Recently, it was performed using a nerve stimulator or ultrasonography [10].

According to the direction of the accessed needle, the classical approach is to vertically come in 2.5 to $3 \mathrm{~cm}$ lateral to the midline. The medial approach is to come in $1 \mathrm{~cm}$ laterally, and paravertebral-peridural block is to approach $45^{\circ}$ on the coronal plane [9], in concern with the frequency of injection, satisfactory results were obtained in more than $90 \%$ by a single injection [11], and it has been also reported that in comparison to single injection, success rate was noticeably high in multilevel injection [12].

In a study conducted on patients undergoing thoracotomy, regarding postoperative pain control during the postoperative period, TPVB was superior to TEB. Pulmonary function tests utilizing the peak expiratory flow rate and oximetry similarly showed better results [13]. Complications may develop in both TEB and TPVB. However, TEB performed in the epidural space can result in catastrophic injuries such as spinal cord injury, and so TPVB may be a safer procedure.

Hypotension or urinary retention due to the blocking of the sympathetic chain may occur in both TEB and TPVB, but the incidence is higher in TEB. Postoperative nausea, vomiting, and respiratory depression are more frequent in TEB than TPVB. In addition, the success rate of TPVB is higher [14]. When comparing bupivacaine with ropivacaine use in breast surgery, cases using ropivacaine showed better results regarding the satisfaction of the patient after surgery, and the continued analgesic period [15].

The authors performed TPVB that is hemodynamically safer and produce fewer complications in comparison with TEB. To raise blockage success rates, multilevel injection was performed, and ropivacaine, which is known for its excellent effects in the aspect of longer analgesic time, was used.

In summary, the authors performed TPVB in a woman 8 weeks pregnant considering the risk/benefit ratio and the effects of anesthetics on the mother and the fetus. Without complications, the breast abscess was safely and successfully removed.

\section{References}

1. Manley S, de Kelaita G, Joseph NJ, Salem MR, Heyman HJ. Preoperative pregnancy testing in ambulatory surgery. Incidence and impact of positive results. Anesthesiology 1995; 83: 690-3.

2. Jenkins TM, Mackey SF, Benzoni EM, Tolosa JE, Sciscione AC. Nonobstetric surgery during gestation: risk factors for lower birthweight. Aust N Z J Obstet Gynaecol 2003; 43: 27-31.

3. Van De Velde M, De Buck F. Anesthesia for non-obstetric surgery in the pregnant patient. Minerva Anestesiol 2007; 73: 235-40.

4. Cohen-Kerem R, Railton C, Oren D, Lishner M, Koren G. Pregnancy outcome following non-obstetric surgical intervention. Am J Surg 2005; 190: 467-73.

5. Howland RH. Categorizing the safety of medications during pregnancy and lactation. J Psychosoc Nurs Ment Health Serv 2009; 47: 17-20.

6. Lane GA, Nahrwold ML, Tait AR, Taylor-Busch M, Cohen PJ, Beaudoin AR. Anesthetics as teratogens: nitrous oxide is fetotoxic, xenon is 
not. Science 1980; 210: 899-901.

7. Wiesner G, Schiewe-Langgartner F, Lindner R, Gruber M. Increased formation of sister chromatid exchanges, but not of micronuclei, in anaesthetists exposed to low levels of sevoflurane. Anaesthesia 2008; 63: 861-4.

8. Cappell MS. Sedation and analgesia for gastrointestinal endoscopy during pregnancy. Gastrointest Endosc Clin N Am 2006; 16: 1-31.

9. Hara K, Sakura S, Nomura T, Saito Y. Ultrasound guided thoracic paravertebral block in breast surgery. Anaesthesia 2009; 64: 223-5.

10. Karmakar MK. Thoracic paravertebral block. Anesthesiology 2001; 95: 771-80.

11. Pusch F, Freitag H, Weinstabl C, Obwegeser R, Huber E, Wildling E. Single-injection paravertebral block compared to general anaesthesia in breast surgery. Acta Anaesthesiol Scand 1999; 43: 770-4.
12. Naja ZM, El-Rajab M, Al-Tannir MA, Ziade FM, Tayara K, Younes F, et al. Thoracic paravertebral block: Influence of the number of injections. Reg Anesth Pain Med 2006; 31: 196-201.

13. Richardson J, Sabanathan S, Jones J, Shah RD, Cheema S, Mearns AJ. A prospective, randomized comparison of preoperative and continuous balanced epidural or paravertebral bupivacaine on post-thoracotomy pain, pulmonary function and stress responses. Br J Anaesth 1999; 83: 387-92.

14. Conlon NP, Shaw AD, Grichnik KP. Postthoracotomy paravertebral analgesia: will it replace epidural analgesia? Anesthesiol Clin 2008; 26: 369-80.

15. Hura G, Knapik P, Misiolek H, Krakus A, Karpe J. Sensory blockade after thoracic paravertebral injection of ropivacaine or bupivacaine. Eur J Anaesthesiol 2006; 23: 658-64. 